\title{
Deviations from Vegard's law in ternary III-V alloys
}

\author{
S. T. Murphy* and A. Chroneos \\ Department of Materials, Imperial College London, London SW7 2AZ, United Kingdom \\ C. Jiang \\ Materials Science and Technology Division, Los Alamos National Laboratory, Los Alamos, New Mexico 87545, USA
}

U. Schwingenschlögl

PSE Division, KAUST, Thuwal 23955-6900, Kingdom of Saudi Arabia

R. W. Grimes

Department of Materials, Imperial College London, London SW7 2AZ, United Kingdom

(Received 17 May 2010; published 3 August 2010)

\begin{abstract}
Vegard's law states that, at a constant temperature, the volume of an alloy can be determined from a linear interpolation of its constituent's volumes. Deviations from this description occur such that volumes are both greater and smaller than the linear relationship would predict. Here we use special quasirandom structures and density functional theory to investigate such deviations for $M_{x} N_{1-x}$ As ternary alloys, where $M$ and $N$ are group III species (B, Al, Ga, and In). Our simulations predict a tendency, with the exception of $\mathrm{Al}_{x} \mathrm{Ga}_{1-x} \mathrm{As}$, for the volume of the ternary alloys to be smaller than that determined from the linear interpolation of the volumes of the $M A$ s and $B A s$ binary alloys. Importantly, we establish a simple relationship linking the relative size of the group III atoms in the alloy and the predicted magnitude of the deviation from Vegard's law.
\end{abstract}

DOI: 10.1103/PhysRevB.82.073201

\section{INTRODUCTION}

Vegard's law is an empirical rule ${ }^{1,2}$ which states that a property value of an alloy (here we will consider the volume) can be determined from a linear interpolation of the property values of its constituent elements, or in the case of higher order alloys, constituent compounds. It is now established that the volume of most alloys cannot be predicted using such an average manner, and that obedience to Vegard's law is more the exception than the rule. ${ }^{3}$ The magnitude of any deviation from the linear interpolation will depend on factors including: (i) the relative size of the constituent elements, (ii) the relative volume per valence electron, (iii) Brillouin-zone effects, and (iv) electrochemical differences between the elements. ${ }^{4}$ An elegant demonstration of the effect of just one of these factors, the relative size of the atoms, is given by Denton and Ashcroft. ${ }^{2}$ The aim of the current work is to: (i) examine the adherence of ternary alloys, formed from two binary group III arsenides, to Vegard's law and (ii) to determine a general relationship between the value of any variation in the covalent radii of the constituent species.

Binary and ternary III-V alloys are a technologically important family of materials. The band gaps of the binary $M X$ alloys can be engineered by doping with other binary $N X$, alloys (where $M$ and $N$ are group III atoms and $X$ is a group $\mathrm{V}$ atom). This has facilitated, for example, the development of highly specific optoelectronic devices such as highefficiency quantum dot (QD) lasers and single or multicolored QD photodetectors made using $\mathrm{In}_{x} \mathrm{Ga}_{1-x}$ As. ${ }^{5}$ Furthermore, complex devices, such as nanosized transistors, can be made by lattice matching layers of binary and/or ternary alloys. ${ }^{6}$ When attempting to lattice match III-V ternary alloys to either binary alloys or other ternary alloys it is important to be able to accurately predict the lattice parameter of the ternary alloy for the relevant composition.
PACS number(s): 61.72.uj

\section{MODELING RANDOM ALLOYS}

The materials examined here all have the formula $M_{x} N_{1-x}$ As where $M$ and $N$ are group III atoms (B, Al, Ga, and In). These materials all exhibit the zinc-blende structure with space group $F \overline{4} 3 m$ (Number 216). For the binary (when $x=0$ or $x=1$ ) III-V alloys (i.e., BAs, AlAs, GaAs, and InAs) the As atoms are assigned to the $4 a$ Wyckoff positions and the group III atoms occupy the $4 c$ Wyckoff sites, thus, creating two inter-penetrating face-centered cubic sublattices. ${ }^{7}$ In the ternary $M_{x} N_{1-x}$ As alloys, occupation of the $4 a$ sublattice by As remains unchanged, however, the two group III ions are distributed randomly on the $4 c$ sublattice.

It is possible to model disordered alloys by applying an effective medium technique, such as the virtual crystal approximation (VCA), ${ }^{8}$ where disorder is represented by assigning appropriate partial occupancies to each site of the disordered sublattice. Unfortunately, this assumes that there is a homogenous distribution of atoms on the disordered sublattice, which ignores the possibility of clustering and associated local distortions. In order to obtain a realistic description of a random alloy, while still retaining full atomistic detail, Zunger et al. ${ }^{9}$ have developed a series of special quasirandom structures (SQS). These quasirandom structures optimize the arrangement of the $M$ and $N$ atoms for a given number of lattice sites, such that the structure as a whole mimics the lowest order correlation functions (pair and many body) of an infinite random alloy. ${ }^{9-11}$ Hass et al. ${ }^{12}$ compared small (16 atom) SQS cells to the simulations of randomly distributed cations in supercells containing very large numbers of atoms $(>500)$ and found that the density-functional theory (DFT) simulations based on the 16 atom SQS supercells adequately reproduced the dominant spectral features observed in the large supercell simulations. As we are not 


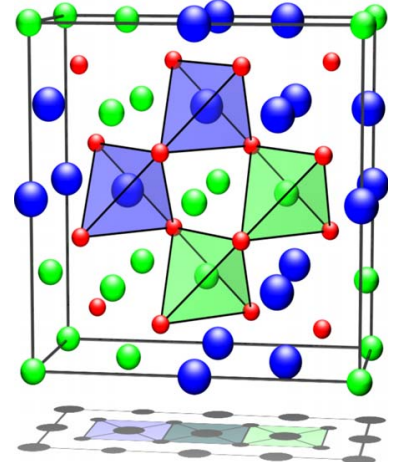

FIG. 1. (Color online) The 32 atom $M_{x} N_{1-x}$ As SQS unit cell where $x=0.5$. This cell is extended in the $c$ direction to form a 1 $\times 1 \times 2$ supercell containing 64 atoms. The red spheres represent As atoms, blue spheres represent $M$ atoms and green spheres represent $N$ atoms.

considering charged defects with large strain fields, minimum image effects are not expected to be an issue, therefore our supercells containing 64 atoms are deemed appropriate for this investigation. This combination of DFT and 64-atom supercells has been successfully implemented by Chroneos et al. $^{13}$

\section{SIMULATION DETAILS}

The simulation SQS supercells adopted here are shown in Figs. 1 and 2 for $x=0.5$ and $x=0.25$ (or 0.75 ) respectively. The cell shown in Fig. 1 contains 32 atoms, however, to create our simulation supercell this cell is extended in the $c$ direction, thus generating a $1 \times 1 \times 2$ supercell. For the simulations where $x=0.25$ or 0.75 the cell shown in Fig. 2 is adopted unchanged.

The simulations were performed within the DFT framework implemented in the CASTEP simulation package. ${ }^{14}$ The structures of our 64 atom SQS $M_{x} N_{1-x}$ As supercells for $x=0,0.031,0.25,0.5,0.75,0.97$, and 1.0 were relaxed to zero strain using energy minimization under constant pressure conditions. Exchange and correlation interactions were modeled using the local density approximation (LDA) and

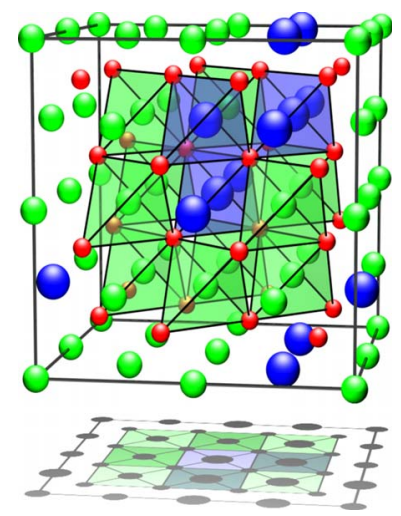

FIG. 2. (Color online) The 64 atom $M_{x} N_{1-x}$ As SQS unit cell for $x=0.75$. The red spheres represent As atoms, blue spheres represent $M$ atoms and green spheres represent $N$ atoms. ultrasoft pseudopotentials with the plane-wave basis expanded to a cutoff of $380 \mathrm{eV}$. Currently, the LDA is the standard for simulations on materials such as GaAs. ${ }^{15,16} \mathrm{~A}$ Monkhorst-Pack ${ }^{17}$ grid of $2^{3} k$-points was used to sample the Brillouin-zone of our 64 atom supercells.

\section{RESULTS AND DISCUSSION}

Figure 3 shows the variation in the unit cell volume, as a function of $x$, for the six random alloys: $\operatorname{In}_{x} \mathrm{Al}_{1-x} \mathrm{As}$, $\mathrm{Al}_{x} \mathrm{~B}_{1-x} \mathrm{As}, \quad \mathrm{Al}_{x} \mathrm{Ga}_{1-x} \mathrm{As}, \quad \mathrm{In}_{x} \mathrm{Ga}_{1-x} \mathrm{As}, \quad \mathrm{Ga}_{x} \mathrm{~B}_{1-x} \mathrm{As}$, and $\mathrm{In}_{x} \mathrm{~B}_{1-x} \mathrm{As}$. The crosses represent the unit cell volumes obtained from the energy minimization of our SQS supercells and the solid line represents the linear interpolation of the end member binary group III arsenide volumes (i.e., Vegard's law). Figure 3 shows that the unit cell volumes for the intermediate compositions of the random alloys deviate from Vegard's law. The magnitude of the deviation $\Delta V(x)$, observed for $M_{x} N_{1-x}$ As can be determined using Eq. (1),

$$
\Delta V(x)=V_{M_{x} N_{1-x} \mathrm{As}}-\left(x V_{M \mathrm{As}}+(1-x) V_{N \mathrm{As}}\right),
$$

where $V_{M_{x} N_{1-x} \text { As }}, V_{M A s}$, and $V_{N A s}$ are the unit cell volumes of $M_{x} N_{1-x} \mathrm{As}, M \mathrm{As}$, and $N \mathrm{As}$, respectively. A positive value of $\Delta V(x)$ corresponds to a ternary alloy with a volume greater than that predicted from Vegard's law and conversely a negative value implies that the volume of the ternary alloy is smaller than the Vegard's law value.
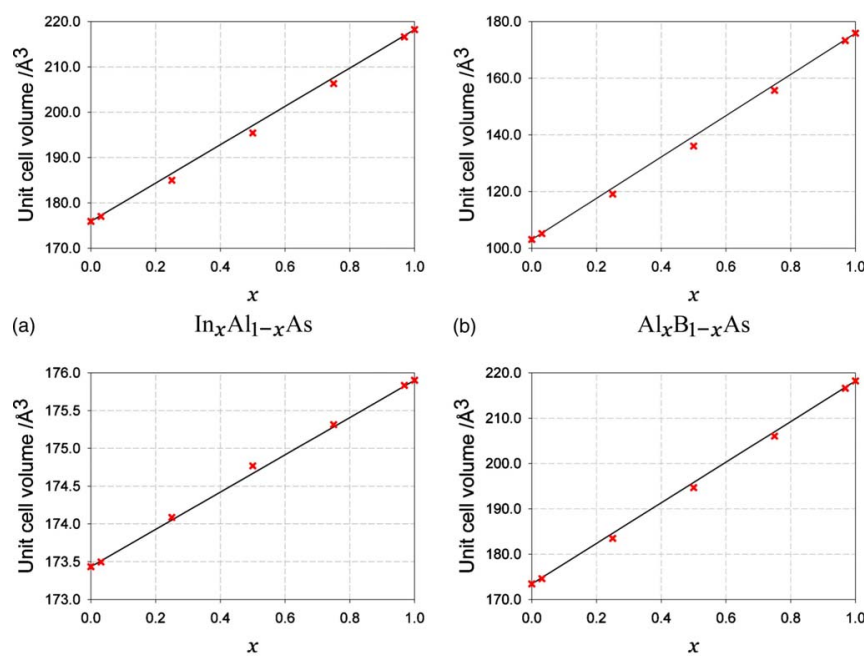

(b)

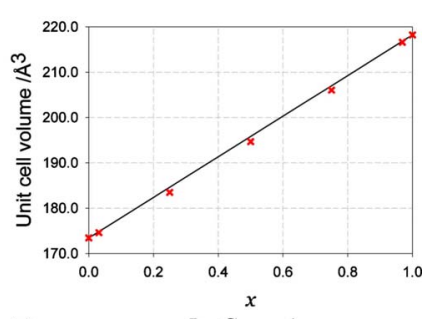

(c)
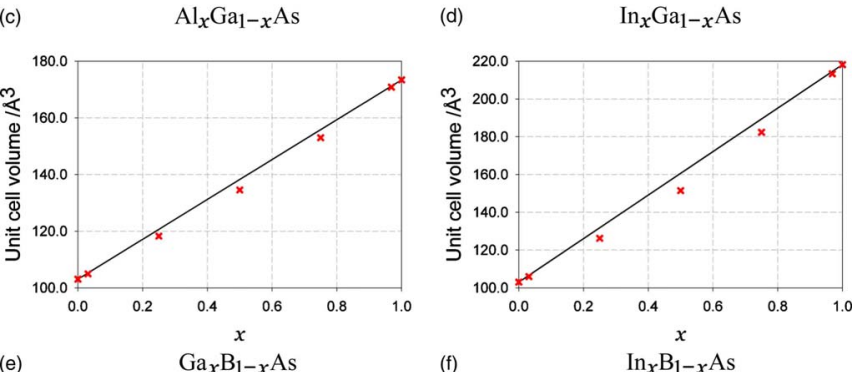

FIG. 3. (Color online) Changes in unit cell volumes as a function of $x$ in $M_{x} N_{1-x}$ As for the six random alloys. The crosses represent the unit cell volumes obtained from DFT simulations and the solid line represents a linear interpolation of the binary alloy volumes (i.e., Vegard's law). 


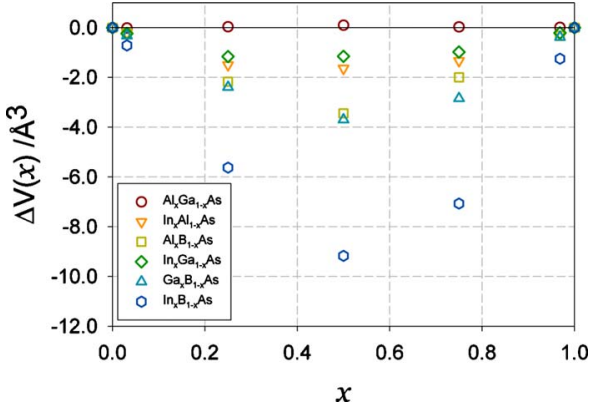

FIG. 4. (Color online) The deviation from Vegard's law $[\Delta V(x)]$ as a function of $x$ in $M_{x} N_{1-x}$ As for the six random ternary alloys.

The deviation from Vegard's law $[\Delta V(x)]$ for each of our random alloys is plotted as a function of $x$ in Fig. 4. Figures 3 and 4 show that for our ternary alloys there is a negative deviation from Vegard's law, with the exception of $\mathrm{Al}_{x} \mathrm{Ga}_{1-x} \mathrm{As}$, which exhibits a very small positive deviation. There is limited experimental evidence concerning such deviations in ternary arsenides. However, for $\mathrm{Al}_{0.5} \mathrm{Ga}_{0.5} \mathrm{As}$, Gehrsitz et al. ${ }^{18}$ predict a deviation of $0.027 \AA^{3}$, which compares favorably with $0.10 \AA^{3}$ predicted by our simulations. Furthermore, Zhou and Usher ${ }^{19}$ also predict a positive deviation for AlGaAs. For $\operatorname{In}_{0.5} \mathrm{Ga}_{0.5} \mathrm{As}$, Katayama et al. ${ }^{20}$ predict a value of $-0.96 \AA^{3}$, compared to $-1.15 \AA^{3}$ obtained from our simulations. Conversely, the empirical based molecular dynamics simulations of Branicio et al. ${ }^{21}$ predict a very slight positive deviation from Vegard's law in $\operatorname{In}_{x} \mathrm{Ga}_{1-x} \mathrm{As}$. This suggests that empirical potential based methods may not be suitable for modeling random semiconductor alloys, as discussed by Chroneos et al., ${ }^{22}$ because they do not include electronic effects.

Chimot et al. ${ }^{23}$ performed LDA simulations on $\mathrm{Ga}_{x} \mathrm{~B}_{1-x} \mathrm{As}$ and $\mathrm{In}_{x} \mathrm{~B}_{1-x} \mathrm{As}$ using the VCA approximation and predict large positive deviations from Vegard's law. As mentioned previously, the VCA assumes a homogeneous distribution of atoms, which ignores local relaxation and therefore treats the alloy as a mean zinc blende structure in which all $4 \mathrm{c}$ sites are identical. By adopting SQS supercells the impact of local environments in providing distorted tetrahedra and alterations to the tetrahedral packing is included into our simula-

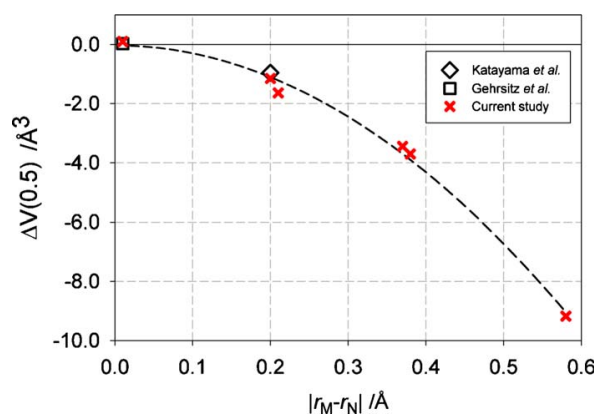

FIG. 6. (Color online) The variation in $\Delta V(0.5)$ as a function of $\left|r_{M}-r_{N}\right|$. The dotted line is the quadratic function $\Delta V(0.5)=a \mid r_{M}$ $-\left.r_{N}\right|^{2}+b\left|r_{M}-r_{N}\right|+c$ where $a=-26.75, b=-0.015$ and $c=-0.034$, with an $R^{2}$ value of 0.99 . For comparison, the experimental data of Katayama et al. (Ref. 20) and Gehrsitz et al. (Ref. 18) have also been plotted.

tions. It is these local distortions (an example of which is shown in Fig. 5) that produce the negative deviation from Vegard's law observed in our simulations.

There have been a number of other studies that examine deviations from Vegard's law in other groups of semiconductor materials. Dridi et al. ${ }^{24}$ examined the deviations in lattice parameters and band gaps of the wurtzite structured ternary $A_{x} B_{1-x} \mathrm{P}$ alloys (where $A$ and $B$ are $\mathrm{Al}, \mathrm{Ga}$, and $\mathrm{In}$ ) and Chizmeshya et $\mathrm{al}^{3}{ }^{3}$ studied the wurtzite group IV $X_{x} Y_{1-x}$ alloys (where $X$ and $Y$ are $\mathrm{Si}, \mathrm{Ge}, \mathrm{Sn}$, and C). The crystallographies of the systems studied by these authors are not the same as the zinc blende structured ternary alloys studied here therefore their observations cannot be compared directly with the present results. However, it is interesting to note the complexity involved in generating an overarching model for predicting deviations from Vegard's law in semiconductors.

Other than the crystal structure type one of the factors known to influence the volume of an alloy is the relative size of the atoms. ${ }^{2}$ To investigate how the relative covalent radii can influence the deviation from Vegard's law, the deviation at $x=0.5$ [i.e., $\Delta V(0.5)]$ is plotted against the absolute difference in the covalent radii of the constituent group III elements (i.e., $\left|r_{M}-r_{N}\right|$ ) in Fig. 6. The covalent radii used here are taken from Cordero et al. ${ }^{25}$ Figure 6 shows that for very small values of $\left|r_{M}-r_{N}\right|$ a very small positive deviation from
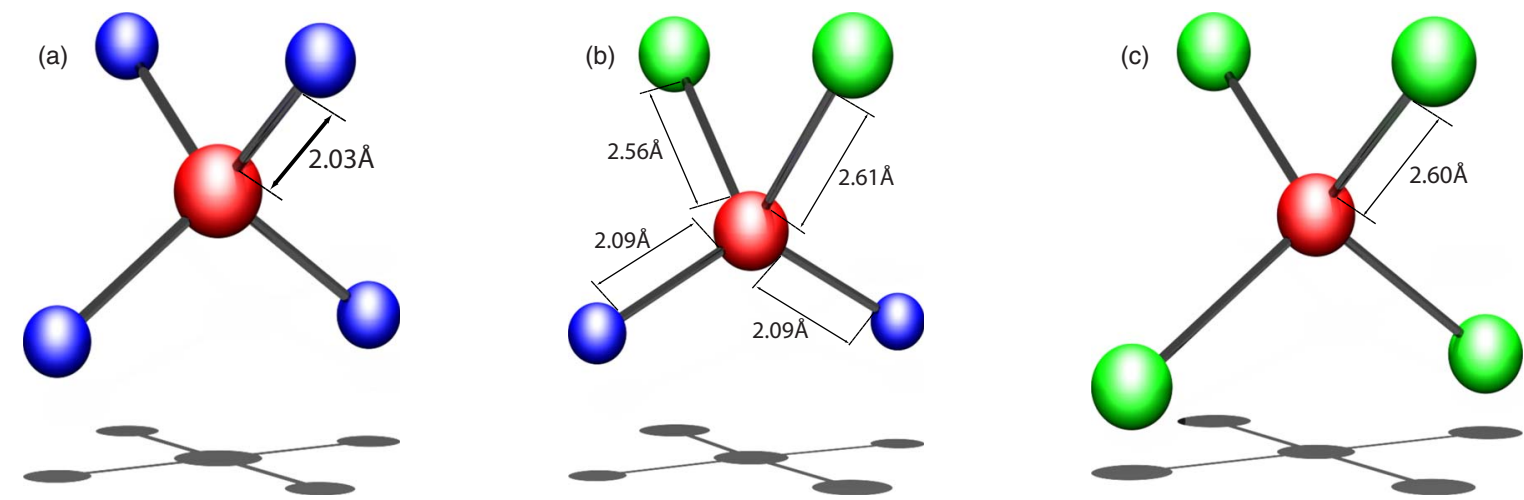

FIG. 5. (Color online) Examples of As lattice sites in: (a) BAs, (b) $\operatorname{In}_{0.5} \mathrm{Ga}_{0.5} \mathrm{As}$, and (c) InAs. In the binary alloys, BAs and InAs, the $\mathrm{B}$ and $\mathrm{In}$ atoms form perfect tetrahedra around the As site, however, in $\mathrm{In}_{0.5} \mathrm{Ga}_{0.5}$ As the bond lengths are not identical and are different from the values in the constituent binary alloys. 
Vegard's law is predicted. Conversely, as the difference in the covalent radii increases the volume of the ternary alloy is predicted to become increasingly smaller than that predicted from Vegard's law. A quadratic function, of the form $\Delta V(0.5)=a\left|r_{M}-r_{N}\right|^{2}+b\left|r_{M}-r_{N}\right|+c$, has been fitted to our data in order to provide a simple predictive model linking the deviation from Vegard's law in ternary arsenides and the absolute difference in the covalent radii of the constituent group III atoms. The parameters for this function are: $a=$ $-26.75, b=-0.015$, and $c=-0.034$ with an $R^{2}$ value of 0.99 . The available experimental data ${ }^{18,20}$ is also reported in Fig. 6 and appears to support the results.

A possible explanation for the very slight positive deviation predicted for $\mathrm{Al}_{x} \mathrm{Ga}_{1-x} \mathrm{As}$ can be found by considering the covalent radii of the group III atoms and the resulting unit cell volumes. The covalent radii for $\mathrm{Al}$ and $\mathrm{Ga}$ are 1.21 and $1.22 \AA$ respectively, ${ }^{25}$ however the values obtained from our simulations predict unit cell volumes of $175.90 \AA^{3}$ for AlAs and $175.43 \AA^{3}$ for GaAs. This shows that despite the covalent radius of $\mathrm{Ga}$ being greater than that of $\mathrm{Al}$ the volume for AlAs is greater than that for GaAs. Therefore, $\mathrm{Al}_{x} \mathrm{Ga}_{1-x} \mathrm{As}$ is the only system examined here where the group III atom with the largest covalent radius does not produce the binary alloy with the largest unit cell volume.

\section{SUMMARY}

We have employed DFT simulations of SQS supercells, to elucidate the relationship between the difference in covalent radii of the constituent group III atoms and the extent of the deviation from Vegard's law, exhibited by $M_{x} N_{1-x}$ As, zinc blende structured, ternary alloys. For the majority of these alloys a negative deviation is predicted (with the exception of $\mathrm{Al}_{x} \mathrm{Ga}_{1-x} \mathrm{As}$ ) and the magnitude of this deviation increases as the difference in the covalent radii of the group III atoms increases. The degree to which this trend can be applied to other ternary alloys such as $M_{x} N_{1-x} \mathrm{P}$ is unknown, however, Geist and Ascheron ${ }^{26}$ calculated a positive deviation from Vegard's law of $0.045 \AA^{3}$ for $\mathrm{Ga}_{0.5} \mathrm{Al}_{0.5} \mathrm{P}$ and a negative deviation of $-0.81 \AA^{3}$ for $\mathrm{Ga}_{0.5} \operatorname{In}_{0.5} \mathrm{P}$. These values are similar to those predicted in our simulations of $M_{x} N_{1-x} \mathrm{As}$, for the same combinations of group III atoms, which encourages us to speculate that this relationship may extend to other zinc blende structured ternary alloys.

\section{ACKNOWLEDGMENTS}

This paper was based on work supported in part by King Abdullah University of Science and Technology (KAUST). C.J. would like to acknowledge funding from the U.S. Department of Energy, Office of Basic Energy Sciences. Computational resources were provided by the Imperial College High Performance Computing Service (Ref. 27). *samuel.murphy@ic.ac.uk

${ }^{1}$ L. Vegard, Z. Phys. 5, 17 (1921).

${ }^{2}$ A. R. Denton and N. W. Ashcroft, Phys. Rev. A 43, 3161 (1991).

${ }^{3}$ A. V. G. Chizmeshya, M. R. Bauer, and J. Kouvetakis, Chem. Mater. 15, 2511 (2003).

${ }^{4}$ H. J. Axon and W. Hume-Rothery, Proc. R. Soc. London A 1, A193 (1948).

${ }^{5}$ V. P. Kunets, T. A. Morgan, Y. I. Mazur, V. G. Dorogan, P. M. Lytvyn, M. E. Ware, D. Guzun, J. L. Shultz, and G. J. Salamo, J. Appl. Phys. 104, 103709 (2008).

${ }^{6}$ D.-M. Lin, C.-C. Huang, and Y.-J. Chan, IEEE Trans. Electron Devices 56, 2638 (2009).

${ }^{7}$ S. Massidda, A. Continenza, A. J. Freeman, T. M. de Pascale, F. Meloni, and M. Serra, Phys. Rev. B 41, 12079 (1990).

${ }^{8}$ C.-J. Yu and H. Emmerich, J. Phys.: Condens. Matter 19, 306203 (2007).

${ }^{9}$ A. Zunger, S. H. Wei, L. G. Ferreira, and J. E. Bernard, Phys. Rev. Lett. 65, 353 (1990).

${ }^{10}$ P. Venezuela, G. M. Dalpian, A. J. R. da Silva, and A. Fazzio, Phys. Rev. B 64, 193202 (2001).

${ }^{11}$ C. Jiang, C. Wolverton, J. Sofo, L. Q. Chen, and Z. K. Liu, Phys. Rev. B 69, 214202 (2004).

${ }^{12}$ K. C. Hass, L. C. Davis, and A. Zunger, Phys. Rev. B 42, 3757 (1990).

${ }^{13}$ A. Chroneos, C. Jiang, R. W. Grimes, U. Schwingenschlögl, and H. Bracht, Appl. Phys. Lett. 95, 112101 (2009).

${ }^{14}$ M. D. Segall, P. J. D. Lindan, M. J. Probert, C. J. Pickard, P. J.
Hasnip, S. J. Clark, and M. C. Payne, J. Phys.: Condens. Matter 14, 2717 (2002).

${ }^{15}$ F. El-Mellouhi and N. Mousseau, Phys. Rev. B 71, 125207 (2005).

${ }^{16}$ F. El-Mellouhi and N. Mousseau, Physica B 401-402, 658 (2007).

${ }^{17}$ H. J. Monkhorst and J. D. Pack, Phys. Rev. B 13, 5188 (1976).

${ }^{18}$ S. Gehrsitz, H. Sigg, N. Herres, K. Bachem, K. Kohler, and F. K. Reinhart, Phys. Rev. B 60, 11601 (1999).

${ }^{19}$ D. Zhou and B. F. Usher, J. Phys. D 34, 1461 (2001).

${ }^{20}$ I. Katayama, T. Nakai, T. Inomoto, and Z. Kozuka, Trans. Jpn. Inst. Met. 30, 354 (1989).

${ }^{21}$ P. S. Branicio, J. P. Rino, F. Shimojo, R. K. Kalia, A. Nakano, and P. Vashishta, J. Appl. Phys. 94, 3840 (2003).

${ }^{22}$ A. Chroneos, H. Bracht, C. Jiang, B. P. Uberuaga, and R. W. Grimes, Phys. Rev. B 78, 195201 (2008).

${ }^{23}$ N. Chimot, J. Even, H. Folliot, and S. Loualiche, Physica B 364, 263 (2005).

${ }^{24}$ Z. Dridi, B. Bouhafs, and P. Ruterana, Semicond. Sci. Technol. 18, 850 (2003).

${ }^{25}$ B. Cordero, V. Gómez, A. E. Platero-Prats, M. Revés, J. Echeverría, E. Cremades, F. Barragán, and S. Alvarez, Dalton Trans. 2008, 2832.

${ }^{26}$ V. Geist and C. Ascheron, Cryst. Res. Technol. 19, 1231 (1984).

${ }^{27} \mathrm{http} / / / \mathrm{www}$.imperial.ac.uk/ict/services/teaching-andresearch services/highperformancecomputing 